\title{
FUNCION SIGNIFICATIVA Y DIFERENCIAL DE LA VOCAL NEUTRA EN EL CATALAN DE BARCELONA
}

Objelivo

I. Es bien sabido que la $a$ y la $e$ átonas se confunden, en la pronunciación barcelonesa, en la llamada "vocal neutra" ${ }^{1}$. Se trata de un rasgo que caracteriza todo el catalán oriental (catalán central, rosellonés, balear), pero que no alcanza al resto del dominio lingüístico (catalán occidental, valenciano). Precisanente por ello, uno de los criterios tradicionales para distinguir entre dialectos orientales y dialectos occidentales ha sido el de la presencia de [ə] en aquéllos y su ausencia en éstos. Pero, por más que no sea un sonido universal en catalán, se le considera muy típico de la lengua, por quedar mejor diferenciadas, con él, las lenguas catalana y castellana (ya que ésta posee una tensión articularia media que no permitiría nunca una pronunciación parecida) ${ }^{2}$, y por la importancia que, de hecho, tiene, en catalán, el habla de Barcelona, una de las que poseen la mencionada vocal neutra.

2. Los lingüistas se han preocupado de una manera especial del carácter fonético de la vocal neutra ${ }^{3}$. Pero muy poco se ha discutido

1 Véase A. BAdía MLARgnRit, Gramditica histórica catalana. Barcelona, I95I, $\S 29$, VIII, pp. 92-93, donde hemos dado algunos detalles sobre este sonido. Para mayor comodidad tipográfica la transcribimos aqui [ə]. Por una razón parecida indicamos la abertura de las vocales con acento grave (è, $\dot{)}), y$ su cerrazón con acento agudo $(\epsilon, \delta)$.

2 Tampoco dejan de distinguirse, en cuanto a esta vocal átona, el catalán y el francés, tanto por las circunstancias en que el souido aparece (hay más casos en francés), como por las peculiaridades de su articulación (posee menor teusión en fraucés).

- B. SCIñDEL, Manual de fonética catalana. Cöthen, I908; J. ARTEAGA PEREIRA, Ullada general a la fonètica catalana. Primer Congrés Internacional de la Llengua Catalana. Barcelona, I908, pp. 445-465; P. BARNILS, Sobre fonètica calalana. Vocals. Bolleti del Diccionari de la Llengua Catalana. Palma, I908-1909, IV, pp. 26 269, 277-282, 293-298 (y ell Anuari de l'Oficina Romànica de Lingüistica i Literalura, 1933, VI, pp. 3-20); P. BARNIIs, Pronunciacions normals calalanes. Anuari de l'Ofic. Rom., I933, VI, pp. I63-I 74 (colección de fragmentos tomados del libro Defectes del parlar, del mismo autor, Barcelona, 1930). 
sobre su papel en el campo significativo y funcional de los sonidos catalanes ${ }^{1}$. Emilio Alarcos Llorach, a quien tanto deben los estudios de fonemática española, trató por primera vez de fonemas vocálicos catalanes ${ }^{2}$. Pues bien, al preguntarse sobre el presunto carácter fonemático de la vocal neutra catalana, el propio Alarcos contesta negativamente con estas palabras: "Ën ningún modo podemos considerar [1a vocal neutra] como fonema distinto de los ya indicados [o sea, de $|a|,|\varepsilon|,|\varepsilon|$ ], puesto que en catalán central sólo aparece [a] en los casos en que ni $|a|$ ni $|\varepsilon|$ ni $|e|$ son posibles, es decir, es sólo la variante de realización de estos fonemas asociada a posición átona. Compárense las realizaciones tónica y átona en las palabras siguientes:

$$
\begin{array}{ll}
\text { paga } & \text { [pagə] - pagar [pəgá] } \\
\text { créixer } & \text { [kręšə]-creixem [krəšęm] } \\
\text { plega } & \text { [plęgə]-plegar [pləgá] "' } 3
\end{array}
$$

Evidentemente, el sonido [ə] es, en los casos citados, y, casi podríamos decir que, de una manera normal, la variante de pronunciación de $|a|,|\varepsilon| \circ|e|+$ "posición átona". Pero hay otros casos en los que falla la "asociación a posición átona" de uno de aquellos tres fonemas: hay casos en que la pronunciación de [ə], es, diriamos, esencial, para ser comprendida la palabra. He aquí, pues, to que nos proponemos presentar ell estas páginas. Daremos, en primer lugar, unos datos para una verdadera conmutación de [ə] ( $\$$ 3-4), seguirán unas consideraciones en torno a los datos presentados ( $\$ 5-6)$, y, después de distinguir dos clases de [ə] en la manera como la lengua considera este sonido ( $\$ \$ 7-8)$, intentaremos llegar a una conclusión ( $\$ 9$ ).

1 Nosotros mismos, en la Gram. hist, citada, § 29, XI, p. 93, después de tratar con un cierto detalle de los sonidos vocálicos de la serie media (unas tres páginas), dedicábamos sólo cinco lineas a su caracterización fonemática. $\mathrm{Y}$ de un modo muy poco preciso: "In cuanto a la vocal neutra, también se puede considerar, probablemente, como fonemar (id., id.).

2 E. ALARCOS LIORACH, Sistemza fonemático del catalán, Archivutm, I953, III, pp. 135-1 46 . El mismo autor lia vueito a ocuparse de vocales, ahora desde un punto de vista diacrónico, en La constitución del vocalismo catalan, Studia Philologica, Homenaje ofrecido a Damaso Alonso. I, Madrid, 1960, pp. 35-49.

- Is. Ar,Arcos LloRACII, Sistema, art. cit., p. 138 . Inn el segundo artículo citado en la nota precedente, dedicado, como hemos diclio, a la evolución de las vocales, no se trata de una manera especial de las posibilidades de que [ə] tenga valor fonemático; pero, de heclio, al decirnos que [ə] resulta de la confluencia, en posición atona, de $|a|=|\varepsilon|=|!|($ (b. 48), Alarcos se ratifica en su primer punto de vista. 
3. Presentamos a continuación una treintena de casos de monosilabos átonos cuyo núcleo es [ə], y que se oponen a otros tantos vocablos $\sin [\partial]$, es decir, con otras vocales. La única diferencia significativa entre todos ellos se encuentra en las vocales, de modo que, si en las palabras con [ə], que encabezan cada serie, sustituimos esta vocal por cualquier otra, queda afectada la significación de la palabra. Recuérdese que, por mucho énfasis que se ponga en la articulación, la vocal neutra [ə] no deja nunca de pronunciarse como neutra ${ }^{1}$, hasta el punto de que, si esto ocurriese, no sería comprendida la palabra. Veamos, pues, los monosilabos con [ə], opuestos a sus correspondientes con otras vocales:

I) a [ə] 'a, en'. Se opone a: ha [á] 'ha' (verbo haver); ah! [á] 'ah' (interj.); $e l$ [è] 'eh' (interj.); he [é] (pero pronunciado corrientemente [i] cn el grupo fónico) 'he' (verbo haver); $i$ [i] ' $y$ ' (conjunción copulativa); $h i$ [i] (adverbio pronomiual); oh [ò] 'oh' (interj.); $O$ [ò] 'o' (conjunción disyuntiva); $h o[\mathrm{u}]$ ' 10 ' (complemento neutro átono) ${ }^{2}$. Se opone, además, a los nombres de las vocales fundamentales de la lengua: $a, e, i, 0, u$ (pronunciadas en barcelonés: [a], [e] , [i], [ò], [u]).

2) de [də] 'de'. Se opone a: dar [dá] 'dar' 3; de [dé] 'nombre de la letra D'; dir [dí] 'decir'; do [dò] 'don'; dó [dò] 'dé' 4; 'do' [dó] (nota musical, a veces se pronuncia [dò] con este último significado); dur [dú] 'duro' (adjetivo), 'llevar' (infinitivo).

3) del [də1] 'del'. Se opone a: dalt [dál] 'arriba'; dol [dòł] 'duelo', 'dolo'; $d u l$ [dúl] 'aullido' (sólo en Ibiza, segúu el $D C V B$ ).

4) dels [dəłs] 'de los'. Se opone a: dalts [dáts] 'las partes altas (de algo)' 5; dols [dòls] 'duelos', 'dolos'; dols [dóls] 'dulce'.

1 A. BAdin MLARgarit, Gram. hist., cit., § 29. VIII, 4), p. 92.

2 Dialectalmente habria que añadir el rosellonés ho ( $<$ HOC), que equivale al adverbio de afirmación si. Citado en el Diccionari Catald Valencid Balear $[=D C V B]$ de ALCOVER-MOLI, Io tomos. I930-1962, s. v. ho.

- Aunque mucho menos usado que donar 'dar', no es desconocida la conjugación de dar (que es, no obstante, defectiva). Dialectalmente, añádase da 'padre' (en el lenguaje de niũos, Menorca) (cit. en el $D C V B$ ).

- Iorma del subjuntivo del verbo donar 'dar' (por la común doni), que aparece cn unos pocos casos, casi siempre expresiones fijas, como $D \dot{e} u$ n'hi dó, Déu nos en do.

- Así en el Diccionari General de la Llengua Catalana, de POMPEU FABRA, segunda ed. Barcelona, I954, s. v. dalt. 
5) el [ə1] 'el' (artículo), 'lo' (pronombre personal); al [ə1] 'al'. Se opone a: alt [á1] 'alto'. Lo mismo en plural: els [ats] 'los' (artículo y pronombre personal); als [əłs] 'a los'. Se oponen a: alts [als] 'altos'.

6) em [əm] 'me'; $a m b$ [əm] ${ }^{1}$ 'con'. Se oponen a: ham [ám] 'anzuelo'; hem [èm] 'hemos'; hem [ém] (expresión de desconfiannza y duda) 2 ; hom [òm] 'uno' (sujeto impersonal, comp. fr. uon"); om [óm] 'olmo'; hum [úm] (expresión de incredulidad y reticencia).

7) en [ən] 'en' (preposición); En (artículo demostrativo personal, masculino); 'de ello', 'de alli' (adverbio pronominal, comp. fr. "en»). Se opone a: han [án] 'han'; on [ón] 'donde'; un [ún] 'un(o)'; unt '3 [ún] 'grasa' 4 .

8) ens [əns] 'nos' (pron. pers.). Se opone a: ans [áns] 'antes', 'sino , que' (adverbio y conjunción); ens [èns]. 'ente'; uns [úns] 'unos'.

9) es [əs] 'se' (pron. personal). Se opone a: as [ás] 'as'; has [ás] 'has' (del verbo haver); ís [és] 'es' (verbo ésser); os [òs] 'hueso'; ós [ós] 'oso'; is [ús] 'uso' (coincide con us 'os', pronombre personal átono). Se opone, además, al plural de los nombres de las vocales fundamentales: les as [ás], les ès [ès], les is [ís], les òs [òs], les us [ús] ${ }^{5}$.

Io) et [at] 'te'. Se opone a et, ut (palabras latinas, en la expresión els ets $i$ uts, et pronunciado con $e$ abierta); $O t$ [òt] 'Otón' (nombre propio).

II) la [lo] 'la' (artículo y pronombre personal, femenino). Se opone a: la [lá] 'la' (nota musical); li [li] 'le' (pronombre personal); l' hi 'se lo' (grupo de pronombres personales átonos); lo [lu] '10' (pron. pers.) 6 .

1 La preposición $a m b$ se pronuncia normalmente [əm]; sólo aparece la $-b$ ante vocal: pa amb oli 'pan con aceite' se pronuncia [pamboli].

2 Generalmente se pronuncia con aspirada inicial: [hem], o reducida a: [mm], pero no es rara la pronunciación dada arriba.

- Se usa muclio wás untet que unt (tienen el mismo significado, según FaBra, Diccionari General, donde se define el segundo por el primero, sin duda para evitar la confusión entre $u n t$ y $u n$, pues ambos se pronuncian igual.

- La palabra ent, que a veces aparece (como equivalente del castellano rente") no existe en catalín. Se la confunde, por castellanismo, con la genuiua ens (comp. uúmero S).

- Añadase host 'lueste', que, si normalmente se pronuncia [dst], puede perder su -t por fonética sintáctica cuando le sigue consonante (A. BADfA MarGarur, Gram. hist., cit., \$ 39. III, pp. II2-I I3).

- Dialectalmente habria que añadir le en la expresión esser le 'ser adecuado' (Mallorca). Cit. en el $D C V B$, s. v. be. 
I2) la'n [lən], l'en [lon] (grupo de los pron. pers. la o el + el adverbio pronominal en). Se opone a: lent [lén] 'lente'; lent [lén] 'lento' (adjetivo); li'n [lin] (grupo del pron. pers. $l i$ ' $1 \mathrm{e}$ ' + el adverbio pronominal en); l'un [lún] 'el uno'.

I3) les [ləs] 'las' (pron. personal). Se opone a: las [lás] 'cansado' (adjetivo); l'as [lás] 'el as'; les [lès] 'leso'; lis [lís] 'lis' (uflor de lis»); l'os [lòs] 'el hueso'; l'ós [lós] 'el oso'; l'us [lús] 'el uso'.

I4) me [mə] 'me' (pron. personal); ma [mə] 'mi' (posesivo proclítico, forma femenina). Se oponen a: mà [má] 'mano'; $m i$ [mi] 'mi' (pron. personal); $m i$ [mí] 'mi' (nota musical); $m$ ' $2 i$ [mi] (grupo del pron. pers. me + el adverbio pronominal $h i$ ); $m \cdot h o$ [mu] (grupo del pron. pers. $m e+$ el neutro pronominal ho); mu [mú] 'bramido' (de ganado bovino) ${ }^{1}$.

I5) me'l [məl] 'me 10', Se opone a: mal [mál] 'mal'; mel [mèl] 'pómulo'; mel [mell] 'miel'; mil [mil] 'mil'; mol [mòl] '(él) muele'; mòlt [mòl] 'molido' 2; molt [mól] 'mucho'; mul [múl] 'mulo'. Lo mismo en plural: me'ls [nəls] 'me los'. Se opone a: mals [máls] 'males'; mels [mèls] 'pómulos'; mels [mèls] 'mieles'; mils [mils] 'miles'; mols [mòls] '(tú) mueles'; mòlts [mòls] 'molidos'; molts [móls] 'muchos'; muls [mułs] 'mulos'.

I6) me'n [mən] (grupo del pron. pers. me + el adverbiopronominal en). Se opone a: mant [mán] 'mucho', 'algun(o)' m'han [mán] 'me han'; ment [mén] 'mente' (sustantivo), 'él) miente' (verbo); món [món] 'mundo'; mont [món] 'monte'; mon [mun] 'ni' (posesivo proclítico, forma masculina); munt [mún] 'montón'.

I7) ne [nə] 'de ello', 'de alli' (adverbio pronominal, comp. fr. (en»); $\mathrm{Na}$ [nə] (artículo demostrativo personal, femenino). Se opone a: 'nar (aféresis de anar) [ná] 'ir'; n'he [né] ${ }^{3}$ (grupo del adverbio pronominal +

1 Dialectalmente se podrían añadir varios casos (citados todos en el $D C V B$, s. v.): 1) mè 'perdiguero' (adjetivo aplicado al perro) (NIallorca, Nenorca); 2) mè (de pi) 'piña en forma de copa, antes de abrirse' (en Petra, Mallorca); 3) mè 'embustero' (Menorca); 4) mé 'agua' (en el lenguaje de niũos) (Mallorca); 5) mé (por més 'más') (en varios sitios de Cataluña); 6) mé 'pero' (Rosellón); 7) mu (por ma 'posesivo femenino átono') (Mallorca, Menorca).

2 Este ejemplo en rigor no es válido porque la pronunciación más frecuente que se le da es [mòlt], de forma que la $-t$ se percibe con claridad, precisamente para distinguirlo de mol '(él) muele', citado antes; en plural si que es correcto: mòlls [mols].

- I'ronunciado comúnmente $n^{\prime} h i$ : n'he fet una 'he hecho una' se pronuncia: [ni fet-unə], pero más correcto es: [ne fet-lulə]. Comp. he (pronunciado hi) (núm. I). 
verbo haver, $I^{2}$ pers. pres. indic.); $n i$ [ni] 'ni' (conjunción); $n^{\prime} l i i$ [ni] (grupo de los adverbios pronominales $e n+h i$, comp. fr. "en», "yy); no [nó] 'no' (adverbio); $n \iota^{1}$ [nú] 'nudo'; $и u$ [nú] 'desnudo' (fem. nula) ${ }^{2}$.

I8) pel [pəl] 'por el'. Se opone a: pal [pál] 'palo'; pèl [pèl] 'pelo'; pol [pòl] 'polo' 3. Lo mismo en plural: pels [pals] 'por los'. Se opone a: pals [páls] 'palos'; pèls [pèls] 'pelos'; pols [pòls] 'polos'; pols [póls] (masculino: el pols) 'el pulso'; pols [póls] (fem.: la pols) 'el polvo'; pols [póls] (masc. plural: els pols) 'polvos de ciertas sustancias'.

19) per [pər] 'por'. Se opone a: par [pár] 'par' (título en algunos estados; pl. pars); en la locución a la par (término bursátil); part [pár] ‘ 'parte'; perd [pèr] ${ }^{5}$ 'pierde'; port [pòr] 'puerto', 'porte' s; pur [púr] 'puro'?

20) que [kə] 'que'. Se opone a: ca [ká] 'perro'; ca [ká] 'nombre de la letra $\mathrm{K}^{\prime} ; c a$ [ka], contracción de casa ${ }^{8}$; cal [ká], interj. para desmentir (como el cast. "iqué va!n) '; què [kè] 'qué' (pronombre relativo tónico; interrogativo); qui [ki] 'quién' (pronombre relativo; interrogativo); $c u$ [ku] 'nombre de la letra $Q$ '.

2I) se [sa] 'se' (pron. pers.); sa [sa] 'su' (posesivo proclítico, forma

$1 \quad N u$ o $u u s$, indistintamente. In algunas pronumciacioues (local, vulgar, etc.) aparece musc.

2 Dialectalmente se podria añadir $N E$, forma abreviada vulgar del nombre propio Senè (cast. "Senénn) (Mallorca). La forma $N e$ se usa en combinación con la de $N_{0}$ (<Abdó, cast. "Abdónu): sant $N o$ i sant $N e$ (Mallorca) (cit. en el DCVB).

3 Dialectalmente añádase pol [pòl] 'tarima que se coloca encima de la quilla de una embarcación' (Menorca) (cit. en el $D C V B$ ).

- La dental final, caduca en varias situaciones, deja de pronunciarse cuando sigue consonante: part principal [pàr prinsipál]. Para este extremo, y para los de las dos notas siguientes, véase A. BADfa MARGarIr, Gram. hist., cit., § 39, III, pp. III2-II3.

5 Lo mismo que en la nota anterior: perd força [pèr forsə].

- Lo unismo que en la nota anterior: port fluvial [pòr flubiál] ('puerto'); port gratuit [por grotuit] ('porte').

7 Dialectalmente añádase per [pèr] 'cubo para la colada' (en La Selva y Costa Brava) (cit. en el $D C V B$ y en el Diccionari General, de P. FABRA).

8 Siempre seguida del determinativo, y singularmente cuando la denominación personal determinativa es algo larga: a ca la meva germana 'en casa de mi hermana'. Rasgo típico del lenguaje hablado.

- Se usa por lo común precedida de la conjunción $i: i$ cal, pero sola tiene el uismo valor, y no es uada rara. 
femenina). Se oponen a: sà [sá] 'sano'; ça [sá] 'aquí' í ça [sá] galicismo bastante usado '; sé [sé] 'sé' (verbo saber)'; $c e$ [sé] 'nombre de la letra C'; si [si] 'seno', 'si' (nota musical); si [si] 'si' (pron. reflexivo de $3 .^{2}$ persona); $s i$ [si] 'si' (conjunción); si [si] 'sí' (adverbio); s'lii [si] (grupo del pron. personal sc + el adverbio pronominal hii); so [sò] 'sonido'; fo [sò] 'eso', 'lo' (demostrativo neutro); só [só] ${ }^{3}$ 'soy'; s'lo [su] (grupo del pron. personal se + el neutro pronominal $h o)$.

22) se'l [sə1] 'se 10' (se = pron. reflexivo). Se opone a: sal [sál] 'sal'; salt [sál] 'saltó'; cel [sèl] 'cielo'; sol [sòl] 'sol' (astro), 'solo' (adjetivo masculino), 'sol' (nota musical); sòl [sòl] 'suelo' 4; sól [sól] 'sol' (astro) (pronunciación únicamente dialectal) ${ }^{5}$. Lo mismo en plural: se'ls [səls] 'se los'. Se opone a: sals [sáls] 'sales'; salts [sáls] 'saltos'; cels [sèls] 'cielos'; sols [sòls] 'soles' (astro), 'solos' (adj. masc.) (y añádase el adverbio sols [sòls] 'solamente'); sòls [sòls] 'suelos'; sóls [sols] 'soles" (astro) (dialectal).

23) se'm [səm] 'se me'. Se opone a: sam [sám], pronunciación vulgar de salm 'salmo'; sem [sèm] 'marchito'; Sem [sèm] (nombre propio bíblico); cim [sím] 'cima'; som [són]] 'somos' (verbo ésser); som [sóm] 'somero'.

24) $s e^{\prime} n$ [sən]] (grupo del pron. pers. se + el adverbio pronominal en). Se opone a: sant [sán] 'san(to)'; ccnt [sén] 'cien'; sent [sén] '(él) sienlte'; cint [sín] 'cinturón'; son [sòn] 'sueño'; son [sòn] (partícula que precede a un nombre para formar, junto con él, una denominación toponímica) (en Mallorca); són [són] 'son' (verbo ésser); son [sun] 'su' (posesivo proclítico, forma masculina).

I Adverbio usado principalmente en la expresión f̧a com lla 'sea como sea'• Otra locución: fa enrera 'tiempo atrás'.

2 Se trata del fr. "ça", que, formando parte de la locución adverbial con f̧a, aparece en expresiones como: això no va pas com ça 'cso no ra como debiera', no estic gaire com ça 'no estoy bien del todo' (del Diccionari General, de P. FABRA, s. v.).

3 Só es forma menos usada que la común sóc, pero no desconocida ni mucho menos. Véase $\mathrm{I}$. DE B. MOLI, La flexió verbal en els dialectes catalans. Amuari de l'Oficina Romànica de Lingiïistica i Litcratura, 1932, V, p. 24.

- A esta serie de voces homófonas (las cuatro se pronuncian con o abierta) no pucde añadirse solt (fem. solla) 'suclto', porque en este caso la -t se pronuncia: [solt]. Comp. G. Coron, Escisiones lexicales en el dominio lingḯstico calalán, Boletin de la Socicdad Casicllonense de Cullura, 195I, XXVII, pp. I24-126.

- Sol 'astro' se pronuncia con o cerrada, a grandes rasgos, cn la diócesis de Gcrowa. 
25) se'ns [səns] 'se nos' 1. Se opone a: sants [sáns] 'santos'; sans [sáns] 'sanos'; cens [sèns] 'censo'; cents [séns] 'cientos'; sents [séns] '(tú) sientes'; cints [sins] 'cinturones'; sons [sòns] 'sueños' (plural de son) y 'sonidos' (plural de so).

26) sc't [sat] 'se te'. Se opone a: set [sèt] 'siete', 'sed' (sust.), 'nombre del juego de tennis'; sét [sét] 'sido' (participio dialectal del verbo ésser), por el común estat, y por el analógico sigut, muy extendido) ${ }^{2}$; sit [sít] 'nombre de varios pájaros'; sot [sòt] 'hoyo'; sud [sut] 'sur'.

27) te [ta] 'te' (pron. pers.); ta [tə] 'tu' (posesivo proclítico, forma femenina). Se opone a: te [tè] 'té' (inglés stean); té [té] 'él tiene' y 'ten' (imperativo) (verbo tenir), 'nombre de la letra T'; $t$ 'hi [ti] (grupo del pronombre personal te + el adverbio pronominal $h i$ ); to [tò] 'tono'; $t u$ [tu] 'tú'; $t$ ' $h o$ [tu] (grupo del pron. pers. te + el neutro pronominal ho).'

28) te'l [tol] 'te lo'. Sc opone a: tal [tál] 'tal'; tel [tè1] 'bizna'; tul [túl] 'tul'. Lo mismo en plural: te'ls [tals] 'te los'. Se opone a: tals [táls] 'tales'; tels [tèls] 'biznas'; tuls [túls] 'tules'.

29) te'm [təm] 'te me'. Se opone a: Tem [tèm] 'Telmo' (nombre propio de hombre) '; tem [tèm] 'tomillo' 4; tem [tém] 'teme' (del verbo témer); tim [tím] 'equipo seleccionado para una competición deportiva' 5 ; tom [tóm] 'tomo'; Tom [tóm] 'nombre propio' s; tomb [tóm] 'tumbo'.

1 Como se verá, casi funciona como si fuese un aplural» de se'n (núm. anterior), cuando en realidad alli se trataba del adverbio pronominal derivado de INDE, mientras que ahora se trata del pronombre personal derivado de Nos. De hecho, se opone, como veremos, a los plurales del número anterior, cou algunas excepciones. Counp. núun. 31 (y nota).

2 Se encuentra sobre toclo en la Plana de Vic, y en extensas zonas del catalán orieutal y occidental, $y$ es tipico del ibicenco. Véase F. DE B. MoL,L, La flexió verbal, art. cit., p. 23.

3 Contracción (hipocoristica o familiar) de la forma más corriente, Telm.

- Forma tipicamente balear; en Mallorca se pronuncia [təm]. Aunque esta pronunciación corresponde a [è] de Barcelona, esto no nos autorizarla a dar el ejemplo [tèm] de arriba (en Barcelona se emplea farigola por stomillon), si no fuese por la solución menorquina, con vocal abierta.

- Tomado del inglés «team». Registrado en el Diccionari General, de P. FABRA, s. v.

- Fin rigor, es nombre de lombre (procedente de su forma inglesa: asi se ha lablado, en español, de La cabaña del tio Tom, por ejemplo); ahora bien, en Cataluña es muy a menudo nombre propio de perro. 
30) $t e^{\prime} n$ [tən] (grupo del pron. pers. $t c+\mathrm{el}$ adverbio pronominal cn). Se opone a: tan, tant [tán] 'tan(to)'; ten [tén] (imperativo de tenir) '; tint [tín] 'tinte'; Ton [tòn], nombre propio de hombre (forma familiar de Antoni); ton [tun] 'tu' (posesivo proclítico, forma masculina).

3I) te'ns [tans] 'te nos' 2. Se opone a: tants [táns] 'tantos'; tens [tèns] 'tenso' (adjetivo); tens [téus] '(tú) tienes'; tints [tíns] 'tintes'; tons [tòns] 'tonos' (plural de to).

4. Hasta aquí sólo hemos dado ejemplos de [ə] en palabras monosilábicas. Es lo más natural, tratándose de elementos átonos, ya que, por esta misma condición, se pronuncian como proclíticos o como enclíticos de las palabras en que se apoyan. Pero no han de faltar casos de dos sílabas, correspondientes a grupos de pronombres átonos. En efecto: no es raro que, en voces de más de una sílaba, se produzca el cambio de significado con sólo poner o dejar de poner [ə] u otra vocal, de suerte rue la presencia o ausencia de la vocal neutra altera el sentido del vocablo. Claro está que, de hecho, las oposiciones en palabras de más de una sílaba siempre presuponen (por el lado de la que contiene la [ə] diferencial) un grupo de palabras, una de las cuales es un monosílabo átono con [ə], del tipo de los registrados en los números I al 3I (§ 3). Daremos tres muestras:

32) me la [molo] 'me la'. Se opone a: mala [málə] 'mala'; mcla [mèla] 3. ${ }^{a}$ pers. presente de indicativo del verbo melar "hacer miel (las abejas)' (cit. en $D C V B)^{3}$; mola [mòlə] 'muela (de molino)'; mula [mú10] 'mula'. Lo mismo ocurriría en plural, con me les [mə ləs] 'me las', opuesto a males, moles, miles, y a la forma verbal de $2 .^{a}$ persona meles.

33) se la [sə lə] 'se la' (se = pron. reflexivo). Se opone a: sala [sálə] 'sala'; cela [sèla] (él) oculta'; Cila o Sila [sílə], forma familiar y vulgar del nombre propio de mujer Cecilia (cit. en $D C V B$ ); sola [sòla] 'planta (del pie)', 'suela (del zapato)'; sola [sòla] 'sola' (adjetivo, forma feme-

1 Como es sabido, esta forma imperativa ten coexiste con otras dos (tingues, te), pero no por ello es rara, ni menos inusitada.

2 Como se verá, casi funciona como si fuese un "plural" de $t e$ 'n (núm. anterior), cuando en realidad alli se trataba del adverbio pronominal en, y aqui se trata del jronombre personal ens. Isto no impide que de hecho $t e^{\prime} n s$ se oponga casi siempre, como veremos, a los plurales del número anterior. Comp. núu. 25 (y nota).

3 Lin cambio, no lo trac el Diccionari General, de I'. I'sura. 
nina de sol) ${ }^{1}$. Lo mismo ocurriria en plural, con se les [səlas] 'se las', opuesto a sales, soles (sust.) y soles (adj.), y a la forma verbal de $2 .^{\mathrm{a}}$ persona celes.

34) te la [tələ] 'te la'. Se opone a tala [tálə] 'tala'; tela [tèlə] 'tela'; tola [tòlo] 'plancha (generalmente metálica)' 2 . Lo mismo ocurriría en plural con te les [tolos] 'te las', opuesto a tales, teles y toles.

\section{Consideraciones sobre los datos anteriores}

5. Quisiéramos comentar algunos aspectos de los datos anteriores (§§ 3-4). En primer lugar, aclarar que los casos que hemos presentado como de elementos opuestos se podrían alargar mucho más: a los vocablos habituales de la lengua, a los menos corrientes pero con todo conocidos, a los dialectalismos (citados casi siempre en nota), habría que añadir (sin perjuicio de los que se nos hayan podido olvidar) una serie de contracciones por fonética sintáctica que se producen continuamente en la lengua hablada. I'or cjemplo: la situación que se crea por la ausencia de la semiconsonante palatal $[j]^{3}$, unida a la relajación articulatoria de las vocales átonas, y al tempo bastante vivo de la dicción; estos tres factores, típicos de la pronunciación catalana, provocan que, cuando la vocal [i] va seguida de la neutra [ə], ésta desaparezca casi siempre 4:

I) i el teu pare 'y tu padre'. Pronunciado: [il] (\$ 3, núm. 5).

2) si cin fas el favor 'si me haces el favor'. Pron.: [sim] (\$3, núm. 23).

1 Dialectalmente se podria añadir sula [súlə] 'lezna' (herramienta de zapatero), forma algueresa, correspondiente al común alena.

2 Tomado del fr. "tôlen; galicismo citado en $D C V B$, pero no recogido en el Diccionati Gencral, de P. Fabra.

3 A. Badía Miargikir, Gram. hist., cit., § $2 S$, IV, pp. 88-89.

- Iis la prosolia que exigen muchas reces las canciones populares. Para 10 citar mis que un par de cjemplos, recuirdense aquellos versos de L'hereu Riera: l'una en diu a l'altra: - I a tu qui ct traurà? donde se pronuncia: [kit]. A qui ct corresponde una sola nota musical, $\mathrm{y}^{\circ}[\mathrm{kj}$ t] $]$ no serfa genuino: la lengua lo resuelve mediante la clesaparición de la [ə]. O bien: "tant si es vol com si no es vol» (de La Santa Espina, letra de A. Gunneri), donde se pronuncia: [siz] y [noz] (anmque este último caso no es el mismo, se encuentra en la linea de que hablamos). Tanta fuerza tiene el heclio que presentamos, que la vocal absorbida llega a no ser ya la neutra [ə], sino una tónica (del verbo ésser, csencialnente apoyado, por ser el nexo rerbal copulativo): "si és servit» (literalnente: 'si está usted servido'; se dice muy' a menudo, como equivalente del esp. 'servidor de usted', al ofrecer algo (un subordinado), y casos pareci(los), donde se pronuncia [sis]; o, del Ave Maria: "el Senyor is amb Vos" (esp). 'cl señor cs contigo)', donde (por pérdida de la $-r$ final) se pronuncia: [ñoz]. 
3) si en vols més 'si quieres más' [de ello]'. Pron.: [sin] ( $\$ 3$, núm. 24).

4) si ens veuen 'si nos ven'. Pron.: [sins] ( $\$ 3$, núm. 25).

5) si et sembla bé 'si te parece bien'. Pron.: [sit] ( $\$ 3$, núm. 26) ${ }^{1}$.

En segundo lugar, hacer ver el carácter de los datos anteriores. No se trata de palabras rebuscadas, poco conocidas y menos empleadas, como ocurre a menudo en las ejemplificaciones de los granıáticos. Son voces muy corrientes y fundamentales, que aparcen incontables veces en las conversaciones de un solo día. Son, en una palabra, vocablos de índole gramatical, y esto basta: artículos, pronombres personales, preposiciones (y combinaciones en grupo de estos elementos). Precisamente la indole gramatical de los datos manejados, y sus confusiones inevitables, son causa de más de una incorrección del habla corriente: [ə1] tanto corresponde a el (artículo) como a al (preposición + artículo) (comp. § 3, número 5): en la necesidad de distinguir, la lengua ha forjado [ənəi] en el segundo caso (como echando mano de una -n-antihiática: a n'cl). De la misma manera, $a$ ell [ $\supset$ éll] corre el riesgo de que la [ग], cu contacto con cll [éll], desaparczca, absorbida en esa [é] (comp. los casos que acabamos de mencionar); solución de la lengua hablada: [onéll] 'a él' (expresión de $a \mathrm{cll}$, pero con una -n- antihiática: $a$ n'cll), e incluso: [əmbéll] 'a él' (adoptando otra preposición, amb, que propiamente significa 'con'); así, en el habla corriente, amb ell tanto significa 'con él' (y esto es correcto: he vingut anl cll 'he venido con él'), como 'a él' (y esto es eufónico, pero incorrecto: ho he dit amb cll 'se lo he dicho a él', en vez del correcto: ho he dit a ell) (y junto al ya citado, y también incorrecto: ho he dit a $n^{\prime}$ ell) (itanta es la necesidad de evitar el hiato de [ə] con cualquier otra vocal!).

6. Ejemplos: En la conmutación de [ə] hemos aducido muchos rocablos concretos, en oposición con los que contienen la vocal neutra. Ahora quisiéramos presentar algunos de estos ejemplos dentro de un contexto, muy breve, pero suficiente para hacer ver el valor de contraste significativo que tiene la palabra con [ə] y con cualquier otra vocal.

Pal drct [pal drèt] 'palo derecho', se opone a: pcl drct [pəl drèt] 'a campo traviesa', sólo en la pronunciación [a] o [ə] de la primera vocal; evidentemente, en el primer caso [pal] lleva un acento secundario, que

1 Lin las transcripciones fonéticas de cstos cinco casos liemos atendido únicamente a la reducción de [iə] a [i]; no se nos oculta que la $l$ de $i$ el ten es dental, la $m$ de si em fas es labiodental, la $n$ de si en vols es lilabial, la $s$ de si ens veurem es sonora, y la $t$ de si ct scmbla forma, con la s siguicnte, un sonido africado. 
no aparece en el segundo [pəl], y ello ya basta para que la [a] no se convierta en [ə] (comp. § 7).

Pèls fins [pèls fíns] 'pelos finos', se opone a: pels fins [pals fíns] 'por los finos', sólo por la pronunciación [è] o [ə] de la primera vocal (como en el ejemplo anterior).

P'crd força [p̀̀r fòrsa]' 'pierde fuerza', se opone a: per força [par fòrso] 'por fucrza', por la pronunciación de la primera vocal (como en los ejemplos anteriores).

Jo sé què dibuixa [kè] 'yo sé qué dibuja' (o: 'lo que dibuja'), se opone a Jo sé que dibuixa [kə] 'yo sé que dibuja'. Sólo la pronunciación de [kè] o [kə] nos informa de si pensamos una oración de relativo-interrogativa o una oración completiva (con la conjunción que).

Sala baixa [sàlo báśə] 'sala baja', se opone a se l'abaixa [sələbášə] 'se lo baja', únicamente en la pronunciación, [à] o [ə] de la primera vocal.

Ia lista se podría hacer interminable: hom diu 'uno dice' / em dius 'me dice'; un dóna 'uno da' / on dóna 'donde da' / en dóna 'da (de cllo)'; ćs vizl 'es vivo' / cs viu 'se vive', etc., son otros tantos ejemplos en los cuales lo diferencial, lo único diferencial, es la presencia de [ə] o de cualquier otra vocal, con su timbre caracteristico. Aunque hemos citado un ejemplo con una palabra bisílaba (sala baixa / se l'abaixa), es evidente que el núcleo fundamental de los datos aducidos lo forman vocablos monosilabos, tanto porque, en definitiva, las varias sílabas se reducen a una sola (como ya hemos dicho, §4) (en el caso citado, se reducen a se, puesto que la tiene la misma pronunciación en los dos ejemplos en oposición), como porque en los bisílabos cobra'mayor importancia el papel diferencial del acento (comp. $\$ 7$ ).

\section{Valor de la alternancia "tonicidad / atonicidad"}

7. Llegamos con esto a la dificultad principal de nuestro esfuerzo: la que procede del hecho, tan característico del catalán, de que los fonemas $|a|,|\varepsilon|$ y $|e|$, en posición átona, se realizan como [ə]. Ya hemos visto $(\S 2)$ cómo E. Alarcos Llorach se apoya en este hecho para negar todo valor fonemático al sonido [ə]. $Y$ asi es, en principio: todos los monosílabos citados en la conmutación de [ə] ( $\S 3)$, que contienen $|a|$,

1 Sobre la no pronunciación de la -d de perd, seguida de consonante, recuérdese lo diclio antes, $\S 3$, múm. I 9 (y sus tres primeras notas). 
$|\varepsilon|$ o $|e|$ resuelven esta vocal en [ə] en derivados en los que haya perdido su primitiva condición de acentuada. He aquí algunos ejemplos, partiendo de los datos anteriores $(\S 3)$ :

Munosilabos citaclos en el $§ 3$

Derivados con [0]

\begin{tabular}{|c|c|c|c|c|}
\hline dar & (núm. 2) & daré & [dəré] & 'daré' \\
\hline alt & (núm. 5) & altesa & [altèza] & 'alteza' \\
\hline ns & (núm. 8) & entitat & [ontitát] & 'entidad' \\
\hline lent & (núm. I2) & lentitud & [lontitút] & 'lentitud' \\
\hline las & (núm. I3) & lassitud & [ləsitút] & 'lasitud' \\
\hline$i \grave{a}$ & (núm. I4) & manipular & [mənipulá] & 'manipular' \\
\hline ral & (núm. I5) & maligne & [malígne] & 'maligno' \\
\hline$e l$ & (uúm. I5) & melós & [məlós] & 'meloso' \\
\hline icnt & (núm. IG) & mental & [məntál] & 'mental' \\
\hline nent & (11úm. IG) & montia & [montio] & 'mentía' \\
\hline pal & (เมน์ I8) & palct & [polet] & 'palito' \\
\hline il & (11úm. I8) & pelar & [pals & 'pelar' \\
\hline bart & (núm. IQ) & partir & [partí] & 'partir' \\
\hline erd & (núm. Ig) & perdem & [pardèm] & 'perdemos' \\
\hline & (núm. 20) & cani & [kəní] & 'canino' \\
\hline & (núm. 2I) & sanitat & [sənitát] & 'sanidad' \\
\hline sal & (núm. 22) & salina & [salinə] & 'salina \\
\hline salt & (núm. 22) & saltar & [səltá] & 'saltar' \\
\hline cel & (núm. 22) & celestial & [səlastiál] & 'celeste' \\
\hline Sem & (núm. 23) & semitic & [səmítik] & 'semítico' \\
\hline$a n t$ & (núm. 24) & santedat & [səntədát] & 'santidad' \\
\hline ent & (núm. 24) & centenar & [səntəná] & 'centenar' \\
\hline sent & (núm. 24) & scritinn & [santín] & 'sentimos' \\
\hline ens & (núm. 25) & censal & [sənsál] & 'censal' \\
\hline set & (núm. 26) & sctanta & [sətántə] & 'setenta' \\
\hline et & (núm. 26) & assedegat & [əşədəogát] & 'sediento' \\
\hline & (11úm. 27) & tcnim & [taním] & 'tenemos' \\
\hline tel & (núm. 28) & telar & [təlá] & 'telar' \\
\hline tem & (núm. 29) & temem & [təmèm] & 'tememos' \\
\hline $2 n$ & (núm. 30) & tantissim & [təntísim] & 'tantísimo'. \\
\hline$n$ & (núm. 3I) & tensio & [tansió] & 'tensión' \\
\hline
\end{tabular}

8. Vamos viendo que hay que hacer una distinción clara entre las palabras que poseen la vocal neutra [0]; una distinción en dos gru- 
pos: I) Palabras polisilabas, en las que la [ə] es usólo la variante de realización de $|a,| \varepsilon|\circ| e \mid$ asociada a posición átona" (como dice $\mathrm{E}$. Alarcos Llorach, comp. § 2); por eso son polisílabas, porque la variante [ə] presupone desplazamiento del acento hacia otra sílaba, de forma que la antigua tónica (dar, ment, pil, etc.) pase a ser átona (daré, mental, pelar). 2) Palabras monosílabas (o polisílabas, pero reducibles, en definitiva. a un núcleo monosilábico), esencialmente átonas, cuya única manera de manifestarse en la lengua es, forzosamente (por su condición de átonas), con la vocal neutra [ə]. Lo explicaremos con unos ejemplos: imaginemos que alguien pronuncia los tres ejemplos dados, con las primeras vocales distintas (según los primitivos correspondientes): [daré], [mentál], [pelá], en vez de reducirlas a [ə]; lo que se nos ocurrirá será que esa persona habla catalán occidental (en cuyo dominio no existe la vocal neutra), o que pronuncia con afectación libresca (cosa que alguna vez ocurre entre personas poco versadas en la lengua escrita), o, en fin, que no ha dominado totalmente un sustrato castellano originario (rasgo no raro en la primera generación, y aun en la segunda, de inmigrantes no catalancs cu Cataluña). Pero no por la sensación de extrañeza que nos produzca la pronunciación de las tres palabras mencionadas dejaremos de comprender su significado, de modo que asimilaremos aquella pronunciación a la más habitual de [dəré], [məntál], [pəlá]. En cambio, cualquier monosílabo de los que encabezan los datos para la conmutación de $[\ni]$ ( $\{3$, núms. I a $3 I)$, si no mantiene su vocal neutra [ə], no puede ser comprendido por los oyentes: si oímos [pèr fòrsa] ( $\$ 6)$, sólo podremos comprender 'pierde fuerza' y nunca se nos ocurrirá que lo dicho signifique 'por fuerza'. Lo mismo pasaría con los demás ejemplos citados $(\S 6)$, y con los innumerables que podriamos aducir.

Conclusión

9. I a vocal neutra [0] del barcelonés hablado ( $y$, en general, de los dialectos catalanes orientales) es, en la mayor parte de los casos en que ocurre, sólo la variante combinatoria de los fonemas $|a|,|\varepsilon|,|e|$ asociada a posición átona (comp. $§ 2$ ). Pero no parece que esto se pueda decir también de los monosílabos átonos cuya única vocal es precisamente la neutra [ə] ( $\$ 3)$. En efecto, el concepto de asociada (que se da a la realización de aquellos fonemas, al unírseles la atonicidad) presupone una situación de asociabilidad: para que la [ə] resulte de la unión de "vocal anterior o media" + "posición átona" tiene que tener opción, tiene que tener alternativa: la ticne en paga/pagar, créixer / creixem, plega / ple- 
gar (\$2), como la tiene en dar / daré, ment / mental, pil / pelar $(\S 8)(\mathrm{y}$, como decímos, en la mayor parte de casos que se pueden presentar), porque el acento pasa de la primera sílaba (o única) a la segunda (y esta última posición provoca, naturalmente, la atonicidad de la primera): cutonces tenemos esa unión de "articulación anterior o media" + "posición átona" = "vocal neutra [ə]".

Pero los monosilabos recogidos autes $(\S 3)$ no tienen alternativa: no pueden estar asociados a posición átona porque ni siquiera son asociables: si dejan la posición átona no son comprendidos ( $\$ 8$, final), porque dejan de significar lo que significaban, o sea, que pasan a ser otras palabras. Al no poseer ninguna posibilidad de alternancia "tónico/átono", estos monosílabos no pueden presentarse como asociados a una posición átona (asociados lo serán pagar, crcixem, etc., porque otras veces son 110 asociados: paga, créixer, etc.): los monosílabos son irremediablemente álonos. Este rasgo repercute en lo funcional de los sonidos catalanes, da a los monosílabos con [ə] un carácter especial que los distingue de los derivados (ejemplos de derivados: $\S 7$ ).

Iin suma, frente a la mayor parte de casos (en que la [ə] cs mera variante combinatoria de los fonemas $|a|,|\varepsilon|,|e|$ cuando no son tónicos), los monosilabos estudiados aquí constituyen una excepción; al unirse en ellos indefectiblemente la articulación de la vocal neutra y la atonicidad $\sin$ alternativa, csta vocal adquiere una evidente función significativa y diferencial, aunque sea sólo en esta circunstancia concreta.

\section{A. M. Badía miargarit.}

Universidad de Barcelona. 